Submitted to the Annals of Statistics

\title{
SUPPLEMENT TO: CONFIDENCE SETS FOR PERSISTENCE DIAGRAMS
}

\author{
By Brittany Terese Fasy ${ }^{1, *}$, Fabrizio Lecci $^{2, \dagger}{ }^{2}$ Alessandro Rinaldo ${ }^{2,}$, \\ Larry Wasserman ${ }^{2, \ddagger}$, Sivaraman Balakrishnan ${ }^{2}$ and Aarti Singh ${ }^{2}$ \\ ${ }^{1}$ Tulane University \\ ${ }^{2}$ Carnegie Mellon University
}

\section{APPENDIX A: INTRODUCTION TO PERSISTENT HOMOLOGY}

In this supplementary material, we provide additional details about homology, simplicial complexes, and stability of persistence diagrams.

A.1. Homology. Homology is a method for quantifying the topological properties of a set $\mathbb{M}$. Here we give a brief introduction. Following a large number of definitions, we give a simple example (Example 1) that illustrates the main ideas.

Informal Description. Given a topological space $\mathbb{M}$, the $j$-dimensional homology group $H_{p}(\mathbb{M})$ is an abelian group generated by the $p$-dimensional holes in $\mathbb{M}$ (this will be made more precise below). The Betti number $\beta_{p}$ is the rank of $H_{p}(\mathbb{M})$ : the value $\beta_{0}$ counts the number of connected components of $\mathbb{M}$, the value $\beta_{1}$ counts the number of one-dimensional holes, and the value $\beta_{2}$ denotes the number of two-dimensional holes. We write $\mathbb{M} \cong \mathbb{N}$ if the topological spaces $\mathbb{M}$ and $\mathbb{N}$ have isomorphic homology groups for all $p$.

Homology. Now we give some details about (simplicial) homology. As mentioned in the introduction, the homology captures the components, tunnels, and voids- in general, it captures the holes; see Figure 1. Although everything carries through for field coefficients, we will assume that we have coefficients in $\mathbb{Z}_{2}=\mathbb{Z} / 2 \mathbb{Z}$. Let $S$ be a finite set. The reader might want to think of $S$ as a sufficiently dense subset of a topological space $\mathbb{M}$ of interest. Let $K$ be a simplicial complex using vertices in $S$, such as the Čech complex defined above. If $\sigma_{1}, \ldots, \sigma_{N}$ are $p$-simplices, we define a $p$-chain to be the formal sum $C=\sum_{j} a_{j} \sigma_{j}$ where $a_{j} \in \mathbb{Z}_{2}$. Thus, a chain can be thought of simply as a list of simplices: $\sigma_{j}$ is in the chain $C$ iff $a_{j}=1$. The sum of two chains $C+D$ can then be regarded as the symmetric set difference of the two chains. The set of $p$-chains form an abelian group $C_{p}$, with the empty set corresponding to the identity element.

The boundary $\partial_{p} \sigma$ of a simplex $\sigma=\left(x_{0}, x_{1}, \ldots, x_{p}\right)$ is defined to be the sum of its co-dimension one faces, i.e.,

$$
\partial_{p} \sigma=\sum_{i=0}^{p} \sigma_{(-i)},
$$

${ }^{*}$ Research partially supported by NSF Grant CCF-1065106.

${ }^{\dagger}$ Research supported by NSF CAREER Grant DMS 1149677.

${ }^{\ddagger}$ Research supported by Air Force Grant FA95500910373, NSF Grant DMS-0806009. 

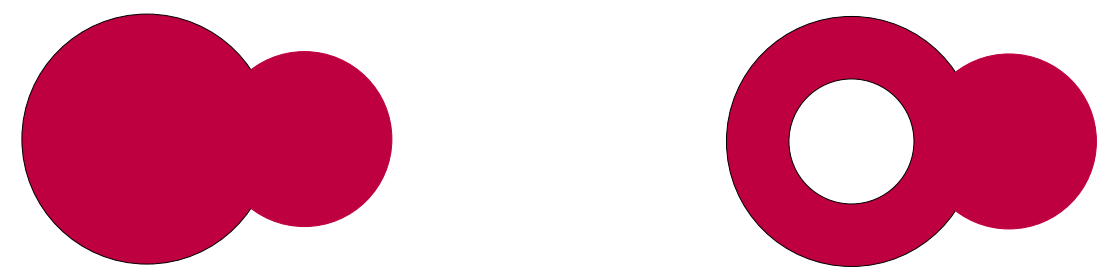

FIG 1. The set on the left has a single connected component. Its zeroth order Betti number is $\beta_{0}=1$. The set on the right also has a single connected component; however, in addition, there is a hole. Its zeroth order Betti number is $\beta_{0}=1$ and its first order Betti number is $\beta_{1}=1$. All other Betti numbers are zero for both sets.

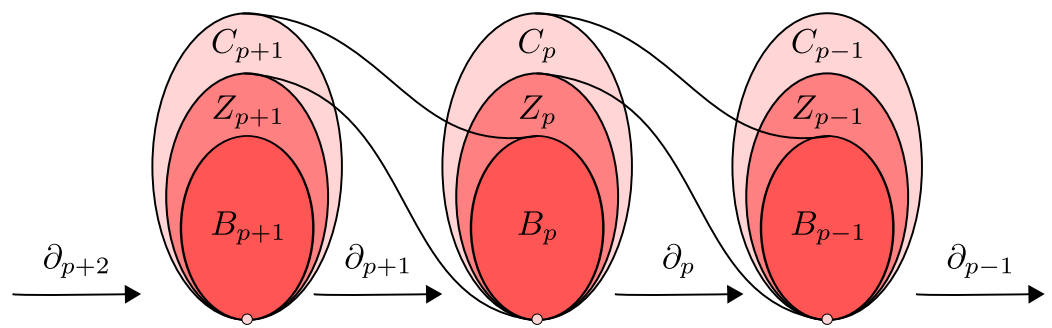

FIG 2. The groups $C_{p}, Z_{p}$ and $B_{p}$ are related by the boundary maps $\partial_{p}$. The $p^{\text {th }}$ homology group $H_{p}$ is the quotient group $Z_{p} / B_{p}$.

where $\sigma_{(-i)}$ is the simplex obtained by removing the vertex $x_{i}$ from $\sigma$. The boundary of a chain $C=\sum_{j} a_{j} \sigma_{j}$ is defined to be $\partial_{p} C=\sum_{j} a_{j} \partial_{p} \sigma_{j}$. It is easy to verify that $\partial_{p} \partial_{p+1} \sigma=0$.

A chain $C \in C_{p}$ is called a cycle if $\partial_{p} C=0$, that is, if the chain has an empty boundary. The set of cycles is a group, denoted by $Z_{p}$. Some elements of $Z_{p}$ are boundaries of chains in $C_{p+1}$. These are called boundary cycles and form a subgroup of $Z_{p}$ denoted by $B_{p}$. In other words, $Z_{p}=\operatorname{ker} \partial_{p}$ and $B_{p}=\mathrm{im} \partial_{p+1}$, where ker and im denote kernel and image. Finally, the homology group is defined to be the quotient group

$$
H_{p}=Z_{p} / B_{p}
$$

The interpretation is that $H_{p}$ is the group generated by equivalence classes of $Z_{p}$, where two cycles $\alpha_{1}$ and $\alpha_{2}$ in $Z_{p}$ are considered equivalent if they differ by the addition of boundary cycles; in other words, if $\alpha_{1}=\alpha_{2}+\alpha_{3}$, where $\alpha_{3} \in B_{p}$. We represent the equivalence class containing $\alpha \in Z_{p}$ as $[\alpha]$; hence, we can write $\left[\alpha_{1}\right]=\left[\alpha_{2}\right]$. If $[\alpha]=[0]$, then we say that $\alpha$ is a trivial cycle; otherwise, $\alpha$ is non-trivial. The Betti number $\beta_{p}$ is the rank of $H_{p}$; that is, $\beta_{p}$ is to the number of generators of (the torsion-free subgroup of) $H_{p}$. The relationships between these groups is summarized in Figure 2. The homology of $\mathbb{M}$, denoted by $\mathcal{H}(\mathbb{M})$ is the collection of these groups $\left\{H_{p}\right\}$.

The following example will clarify the ideas.

Example 1. Consider the dataset in Figure 3. There are 5 points labeled $a, b, c, d$, e. The first plot shows a simplicial complex $\mathcal{K}$ consisting of the following simplices:

$$
\mathcal{K}=\{(a),(b),(c),(d),(e),(a, b),(b, c),(c, d),(d, e),(e, a),(b, d),(b, c, d)\} .
$$


The second plot shows a chain of one-simplices

$$
\alpha_{1}=(a, b)+(b, d)+(d, e)+(e, a)
$$

and the third shows another chain

$$
\alpha_{2}=(a, b)+(b, c)+(c, d)+(d, e)+(e, a) .
$$

We claim that both chains are cycles. To see this, apply the boundary operator:

$$
\begin{aligned}
\partial \alpha_{1} & =\partial(a, b)+\partial(b, d)+\partial(d, e)+\partial(e, a) \\
& =(a)+(b)+(b)+(d)+(d)+(e)+(e)+(a) \\
& =\emptyset
\end{aligned}
$$

where we recall that the sum is taken modulo two. Similarly, $\partial \alpha_{2}=\emptyset$. The fourth plot shows another chain $\alpha_{3}=(b, c)+(c, d)+(b, d)$ and it is easy to see that this is also a cycle. So $\alpha_{1}, \alpha_{2}, \alpha_{3} \in Z_{1}$.

Now $\alpha_{3}$ is not just a cycle, it is also a boundary cycle, because it is the boundary of the triangle $(b, c, d)$ :

$$
\partial(b, c, d)=(b, c)+(c, d)+(b, d)=\alpha_{3} .
$$

Thus $\alpha_{3} \in B_{1}$.

Next, we claim that $\alpha_{1}$ and $\alpha_{2}$ are equivalent cycles. Intuitively, this means that they surround the same hole (the empty square). Formally, these cycles are equivalent because they differ by a boundary cycle:

$$
\alpha_{1}=\alpha_{2}+\alpha_{3}
$$

The set of all first order cycles is $\left\{\emptyset, \alpha_{1}, \alpha_{2}, \alpha_{3}\right\}$. Hence, the first cycle group is: $Z_{1}=\left(\left\langle\alpha_{1}, \alpha_{2}\right\rangle,+_{2}\right)$. The set of boundary cycles is $\left\{\emptyset, \alpha_{3}\right\}$, hence: $B_{1}=\left(\left\langle\alpha_{3}\right\rangle,+_{2}\right)$. The first homology group is

$$
H_{1}=<\left[\alpha_{1}\right]>
$$

where $\left[\alpha_{1}\right]$ represents "the equivalence class of cycles corresponding to $\alpha_{1}$." Since $H_{1}$ is generated by one cycle, we have that $\beta_{1}=1$.

A.2. Simplicial Complexes. Computing the homology directly from $\widehat{S}_{\varepsilon}$ is difficult. Instead, one constructs a set of simplices at scale $\varepsilon$. For example, Figure 4 shows a sequence of Čech complexes (defined below) which is a collection of simplices defined by connecting points at scale $\varepsilon$. These complexes are used to extract the topological features. The Nerve Lemma (see below) guarantees that $\widehat{S}_{\varepsilon}$ and the Čech complex have the same homology.

Complexes. We compute homology using simplicial homology. A simplicial complex $K$ is a set of simplices such that the following two conditions hold:

1. If $\sigma$ is a simplex in $K$ and $\tau$ is a face of $\sigma$, then $\tau$ is also an element of $K$.

2. The intersection of two simplices in $K$ is either a simplex in $K$ or empty. 

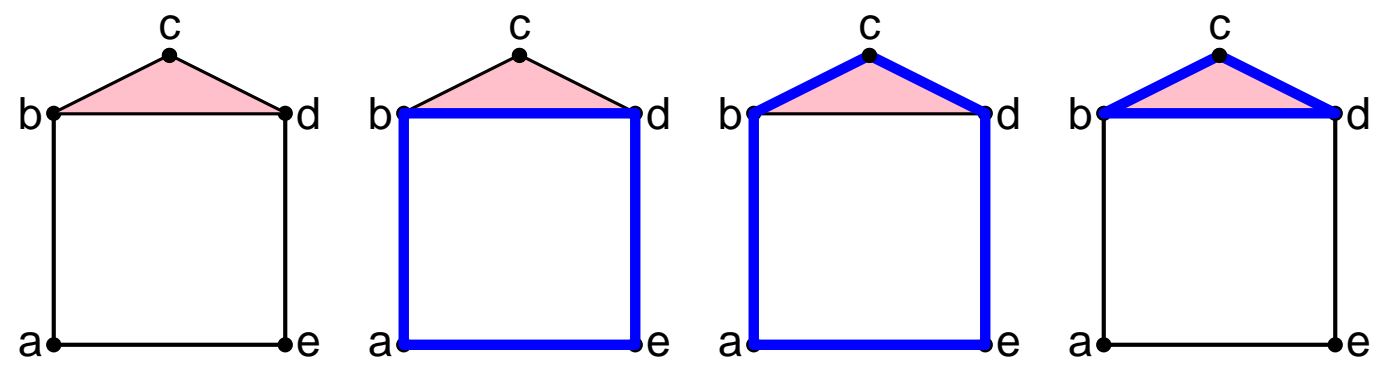

FIG 3. First: A simplicial complex. Second: a cycle $\alpha_{1}$ is highlighted. Third: a different cycle $\alpha_{2}$ that is homologically equivalent to $\alpha_{1}$. Fourth: another cycle $\alpha_{3}$. The cycle $\alpha_{3}$ is a boundary cycle since it is the boundary of the triangle $(b, c, d) . \alpha_{1}$ is equivalent to $\alpha_{2}$ since $\alpha_{1}=\alpha_{2}+\alpha_{3}$. Both cycles $\alpha_{1}$ and $\alpha_{2}$ surround the same hole (the square formed by $a, b, d, e)$.
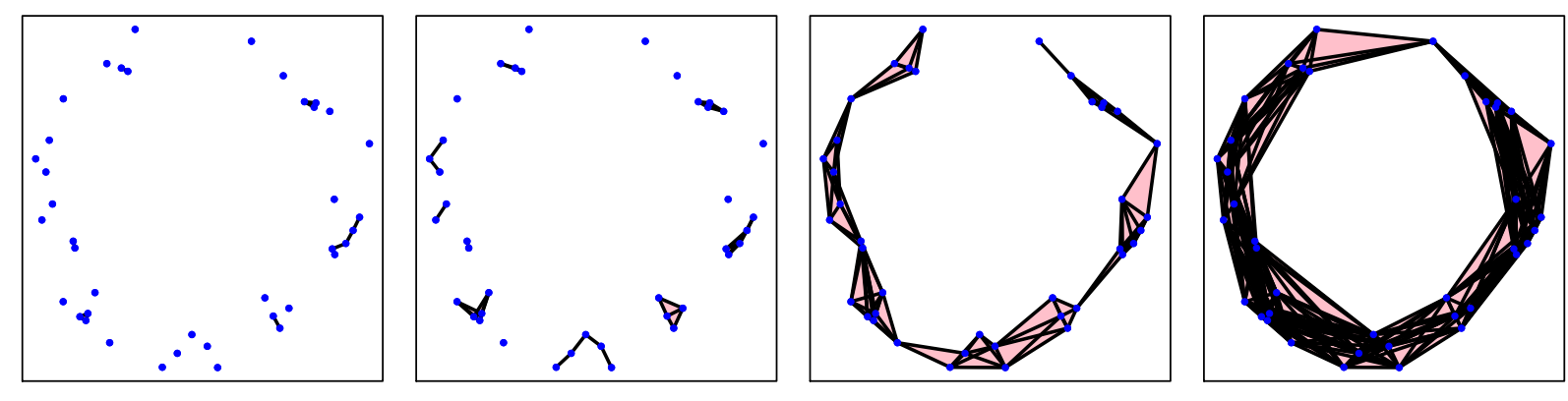

FIG 4. The Čech complex - a collection of simplices - for increasing values of $\varepsilon$. The topological structure of $S_{\varepsilon}$ can be analyzed using the Čech complex. Persistent homology is a method for tracking the topology of $S_{\varepsilon}$ as a function of $\varepsilon$.

The topological space obtained by taking the union of simplices in $K$ under the subspace topology (inherited from the ambient Euclidean space) is denoted by $|K|$.

Often, we are given a set of points $S$, each point belonging to $\mathbb{R}^{D}$, and wish to construct a simplicial complex from them. A natural way to do this is to choose a radius $\varepsilon$ and to center a ball of radius $\varepsilon$ at each point in $S$. The Cech complex is a simplicial complex homotopy equivalent to this union of balls. Homotopy equivalence means that they have similar topological features and, in particular, isomorphic homology groups; see Chapter 0 of Hatcher (2002) for more details.

The Čech complex, denoted by $\check{C} \operatorname{ech}(S, \varepsilon)$, is the set of simplices $\sigma$ with vertices $v_{1}, \ldots v_{k} \in S$ such that

$$
\bigcap_{i=1}^{k} B\left(v_{i}, \varepsilon\right) \neq \emptyset,
$$

where $B\left(v_{i}, \varepsilon\right)$ is the ball of radius $\varepsilon$ centered at $v_{i}$. As stated above, this simplicial complex shares the same homotopy type as the union of the balls centered at the point in $S$. We formalize this statement in the following theorem.

Lemma 2 (The Nerve). The union of balls $S \oplus \varepsilon$ and the Čech complex $\breve{C} \operatorname{ech}(S, \varepsilon)$ are homotopy equivalent. 
It is common to approximate the Čech complex with the Vietoris-Rips complex $V(S, \varepsilon)$, which consists of simplices with vertices in $S$ and diameter at most $2 \varepsilon$. In other words, a simplex $\sigma$ is included in the complex if each pair of vertices in $\sigma$ is at most $2 \varepsilon$ apart. This complex is easier to compute than the Cech complex, because only pairwise distances need to be computed. These complexes satisfy the following sandwich relation:

$$
\check{\operatorname{Cech}}(S, \varepsilon) \subset V(S, \varepsilon) \subset \check{\operatorname{Cech}}(S, \sqrt{2} \varepsilon) .
$$

We note here that, in some literature, the Vietoris-Rips complex comprises of the simplices of diameter at most $\varepsilon$. We prefer the former definition, as then the Vietoris-Rips complex and the Čech complex agree on the one-skeleton for the same parameters.

A.3. Computing and Estimating the Homology of Manifolds. Let $\mathbb{M} \subset \mathbb{R}^{D}$ be a smooth $d$-dimensional manifold and let $m$ be the homological feature size of $\mathbb{M}$, defined as the smallest positive $t$ such that the inclusion $\mathbb{M}_{t / 2} \hookrightarrow \mathbb{M}_{t}$ does not induce an isomorphism on homology. Let $\mathcal{S}_{n}=\left\{X_{1}, \ldots, X_{n}\right\}$ be a set of points sampled from $\mathbb{M}$ such that $\mathbf{H}(\mathcal{S}, \mathbb{M})<m / 4$. We want to use $\mathcal{S}_{n}$ to compute $H(\mathbb{M})$, even though $H(\mathbb{M})$ cannot be computed directly. We proceed in three steps.

First. We would like to expand $\mathbb{M}$ slightly without changing its homology; that is, for $\varepsilon$ small enough, the following equation should hold:

$$
\mathbb{M} \cong \mathbb{M} \oplus \varepsilon
$$

Indeed, this equation holds for $\mathbb{M}$ a smooth sub-manifold of $\mathbb{R}^{D}$ (Chazal and Oudot (2008)).

Second. Let $i$ be the inclusion $\mathcal{S}_{n} \oplus m / 4 \hookrightarrow \mathcal{S}_{n} \oplus 3 m / 4$. Then, the image of the induced map on homology is isomorphic to the homology of $\mathbb{M} \oplus \varepsilon$, for $\varepsilon$ small enough. That is, $\mathbb{M} \oplus \varepsilon \cong \mathcal{S}_{n} \oplus m / 4$ up to features with persistence less than $2 m$ (Cohen-Steiner et al. (2007)). This means that we can compute the persistent homology for $d_{\mathcal{S}_{n}}$ and read off the homology of $\mathbb{M}$, and in particular generators for the homology of $\mathbb{M}$ are also generators for the homology of $\mathcal{S}_{n} \oplus m / 4$. We will denote this relationship with $\cong_{m}^{*}$, as follows:

$$
\mathbb{M} \oplus \varepsilon \cong_{m}^{*} \mathcal{S}_{n} \oplus m / 4 \text {. }
$$

Third. Finally, we need a way to compute $\mathcal{S}_{n} \oplus m / 4$, and we use the Nerve Lemma to obtain:

$$
\mathcal{S}_{n} \oplus m / 4 \cong \operatorname{Cech}\left(\mathcal{S}_{n}, m / 4\right)
$$

We summarize our observations:

$$
\mathbb{M} \cong \mathbb{M} \oplus \varepsilon \cong_{m}^{*} \mathcal{S}_{n} \oplus m / 4 \cong \operatorname{Cech}\left(\mathcal{S}_{n}, m / 4\right)
$$

A.4. Filtrations. The sequence of Čech complexes obtained by gradually increasing the radius of the balls creates a sequence of inclusions. In general, a filtration is a sequence of topological spaces related by (forward or backward) inclusions. In this paper, we will only consider forward inclusions between simplicial complexes. There are two special filtrations that we consider. Let $\mathbb{X}$ be a nice topological space, and let $\mathbb{X}_{s}=d_{\mathbb{X}}^{-1}([0, s])$. If $s<t$, there is a natural inclusion $i_{s, t}: \mathbb{X}_{s} \hookrightarrow \mathbb{X}_{t}$ 
that induces a group homomorphism $i_{s, t}^{*}: H_{p}\left(\mathbb{X}_{s}\right) \rightarrow H_{p}\left(\mathbb{X}_{t}\right)$. The ordered set of complexes $\mathbb{X}_{s}$ as $s$ varies from $-\infty$ to $\infty$ is called the lower level set filtration of $f$. Instead of directly computing the homology of this continuous filtration, we use the Vietoris-Rips filtration:

$$
\left|K_{0}\right| \hookrightarrow\left|K_{1}\right| \hookrightarrow \cdots \hookrightarrow\left|K_{n}\right|
$$

where $K_{i}$ is a Vietoris-Rips complex computed on the input set $S, K_{0}=\emptyset, K_{n}$ is the complete complex on all vertices, and all possible Vietoris-Rips complexes on the input set are represented in this sequence. The justification of using this approximation is given by (2).

In the paper we used the upper level set filtration, which is defined analogously for a function $f$ as the sequence of topological spaces realized as the preimage $f^{-1}(t, \infty)$, and can be approximated for Lipschitz functions by discretizing the domain.

A.5. Persistence Diagrams. Consider a discrete filtration of topological spaces, such as the one given in (5). Applying the homology functor, we obtain a finite sequence of vector spaces:

$$
H_{p}\left(\left|K_{0}\right|\right) \rightarrow H_{p}\left(\left|K_{1}\right|\right) \rightarrow \cdots \rightarrow H_{p}\left(\left|K_{n}\right|\right) .
$$

We say that a homology class $[\alpha]$ represented by a $p$-cycle $\alpha$ is born at $K_{s}$ if $[\alpha]$ is not supported in $K_{r}$ for any $r<s$, but is nontrivial in $H_{p}\left(\left|K_{s}\right|\right)$. The class $[\alpha]$ born at $K_{s}$ dies going into $K_{t}$ if $t$ is the smallest index such that the class $[\alpha]$ is supported in the image of $i_{s-1, t}^{*}$. The birth at $s$ and death at $t$ of $[\alpha]$ is recorded in the $p^{t h}$ persistence diagram $\mathcal{P}_{p}\left(d_{\mathcal{S}_{n}}\right)$ as the point $(s, t)$.

The persistent homology of a function $f$ is defined to be the finite multi-set of pairs of birth-death pairs (along with the infinite set of pairs $(t, t)$ for $t \geq 0$. The persistent homology can be summarized as either a persistence diagram by plotting each pair $(s, t)$ as a point in the extended plane, or a barcode plot by adding an interval for each pair.

A.6. Bottleneck Distance $\boldsymbol{W}_{\infty}$. We now define a metric $W_{\infty}$, called the bottleneck distance, that measures the distance between two persistence diagrams. Intuitively, the bottleneck distance between two persistence diagrams is the maximum distance between points on the first diagram and points on the second diagram, after matching the points optimally; see Figure 5.

We first describe the general matching problem, where we match the elements of a set $A \subseteq \mathbb{R}^{2}$ with elements of a second set $B \subseteq \mathbb{R}^{2}$. A matching between $A$ and $B$ is a set of edges $(a, b)$ with $a$ in $A$ and $b$ in $B$ such that no vertex is incident to two edges. A matching is maximal if the addition of any edge would result in a graph that is no longer a matching. A matching is perfect if every vertex is incident on exactly one edge. In other words, it is a matching where there does not exist an unmatched vertex.

Letting $f, g: \mathbb{M} \rightarrow \mathbb{R}$, the goal is to obtain a perfect matching between the points in $\mathcal{P}(f)$ and in $\mathcal{P}(g)$ that minimizes the cost associated with the matching. To resolve the issue where the number of off-diagonal points in both diagrams is not equal or the diagrams are dissimilar, we allow an off-diagonal point to be matched to a point on the line $y=x$. Hence, we let $A$ be the set of off-diagonal points in $\mathcal{P}(f)$ along with the orthogonal projection of the off-diagonal points in $\mathcal{P}(g)$ onto the line $y=x$. Symmetrically, we let $B$ be the set of off-diagonal points in $\mathcal{P}(g)$ along with the orthogonal projection of the off-diagonal points in $\mathcal{P}(f)$ onto the line $y=x$. The cost of a matching 


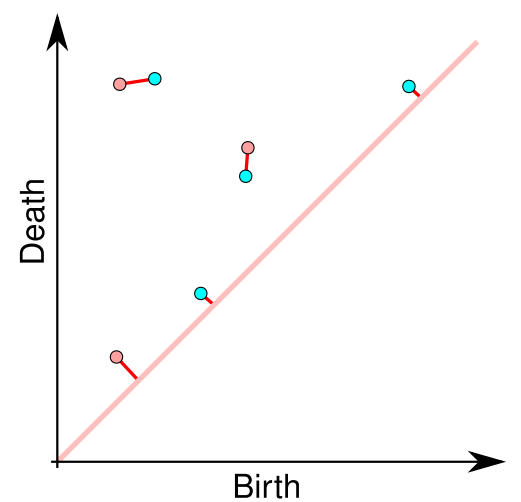

FIG 5. Two persistence diagrams are overlaid. The matching between the two diagrams is indicated by adding a line segment between paired points in the persistence diagrams. In general, a persistence diagram consists of a finite number of off-diagonal points as well as the diagonal $y=x$. Hence, when we take the pairing, we allow a point to be paired with a point on the diagonal, as shown above.

is a function of a cost of the edges in the matching. The cost $d_{\infty}(a, b)$ between points $a$ and $b$ of the persistence diagram $\mathcal{P}(f)$ is the $L_{\infty}$-distance:

$$
d_{\infty}(a, b)=\max \left\{\left|a_{x}-b_{x}\right|,\left|a_{y}-b_{y}\right|\right\}
$$

where $\left(a_{x}, a_{y}\right)$ and $\left(b_{x}, b_{y}\right)$ are the coordinates of $a$ and $b$. As we will see next, the bottleneck distance minimizes the maximum pairwise cost. The bottleneck matching minimizes the bottleneck cost function, which is defined as follows:

Definition 3 (Bottleneck Cost). The bottleneck cost of a perfect matching $P$ is the maximum edge cost in the matching:

$$
C_{B}(P)=\max _{(a, b) \in P} d_{\infty}(a, b)
$$

We minimize this cost over all perfect matchings of $A$ and $B$ to obtain the bottleneck distance between the two sets:

$$
W_{\infty}(A, B)=\min _{P} C_{B}(P)
$$

The matching that attains the bottleneck distance is called the bottleneck matching. Thus, if $f$ and $g$ are two functions and $\mathcal{P}(f)$ and $\mathcal{P}(g)$ are the corresponding persistence diagrams, then we define the bottleneck distance between the diagrams

$$
W_{\infty}(\mathcal{P}(f), \mathcal{P}(g))
$$

as the above minimum cost of the best matching.

\section{REFERENCES}

Chazal, F. and Oudot, S. Y. (2008). Towards persistence-based reconstruction in euclidean spaces. In Proceedings of the twenty-fourth annual symposium on Computational geometry. ACM, 232-241. 
Cohen-Steiner, D., Edelsbrunner, H. and Harer, J. (2007). Stability of persistence diagrams. Discrete Comput. Geom., 37 103-120.

Hatcher, A. (2002). Algebraic topology. Cambridge.

B.T. FASY

Computer Science Department

Tulane University

New Orleans, LA 70118

E-MAIL: brittany.fasy@alumni.duke.edu

\author{
S. BALAKRISHNAN \\ A. Singh \\ Computer Science Department \\ Carnegie Mellon University \\ Pittsburgh, PA 15213 \\ E-MAIL: sbalakri@cs.cmu.edu \\ E-MAIL: aarti@cs.cmu.edu
}

\author{
F. LECCI \\ A. Rinaldo \\ L. WASSERMAN \\ Department of Statistics \\ Carnegie Mellon University \\ PitTsburgh, PA 15213 \\ E-MAIL: lecci@cmu.edu \\ E-MAIL: arinaldo@cmu.edu \\ E-MAIL: larry@cmu.edu
}

